\title{
Development of nanosponges from erythrocyte ghosts for removal of streptolysin-O from mammalian blood
}

\begin{abstract}
Aim: To produce mammalian biomimetic nanosponges from mammalian erythrocyte ghosts. Biomimetic nanosponges were studied in vitro as treatment platforms against exotoxin-related sepsis. Methods: Ovine blood was treated with hypotonic buffer to create erythrocyte ghosts and then subjected to sonication to produce erythrocyte vesicles of nonuniform size. Vesicles were then serially extruded through $400-\mathrm{nm}$ and $100-\mathrm{nm}$ polycarbonate membranes. Nanosponges were prepared by fusing poly(D,L-lactic-co-glycolic acid) cores with ovine erythrocyte vesicles. Results: Ovine erythrocytes were the most susceptible to streptolysin-O lysis, making it a model to study sepsis treatment. Ovine nanosponges adsorbed streptolysin- $\mathrm{O}$ at 37 and $40^{\circ} \mathrm{C}$. Conclusion: These results identify ovine nanosponges as novel therapeutic model to test adsorption of cholesterol binding toxins such as streptolysin-O.
\end{abstract}

First submitted: 25 April 2016; Accepted for publication: 27 June 2016; Published online: 21 October 2016

Keywords: biomimetic $\bullet$ erythrocyte ghost $\bullet$ nanoparticle $\bullet$ nanosponges $\bullet$ streptolysin-O

- toxin absorption

Bacteria can invade and destroy human cells due to virulence factors known as endotoxins and exotoxins. These virulence factors cause a condition known as sepsis (systemic inflammatory response syndrome), which is a whole body inflammation. Severe sepsis (organ dysfunction) or septic shock (abnormally low blood pressure and organ damage) is a major public health problem in England [1]. From 2001 to 2010 , there were 226,547 associated deaths. About $50 \%$ of sepsis cases can be attributed to bacterial pathogens [2]. Sepsis was observed to be directly related to age with a positive correlation between increasing numbers of deaths and age. To reduce the mortality rate caused by sepsis, there has to be an improvement in treatment.

Common toxins associated with sepsis are pore-forming toxins (PFTs). PFTs are proteins released by bacteria that are widely distributed and form lesions in the host cellular membrane. The toxins found to be most potent are $\alpha$-hemolysin and streptolysin-O. $\alpha$-hemolysin is released by Staphylococcus aureus and streptolysin-O is released by Streptococcus pyogenes [3]. These toxins are released in the blood stream of an infected patient and bind to a variety of cells including red blood cells (RBCs) and cause them to hemolyze [4].

\section{Mechanism of PFT binding}

The principal function of pore-forming toxins is to disrupt host cell membranes. This function is carried out to kill target cells, to release nutrients and, at times, delivery of bacterial or external factors. Loss of this function could cause the pathogenic strain to become less virulent. PFTs are generally secreted as a water soluble monomer. These toxins tend to be specific for cellular targets on cellular membranes; upon recognition they bind to the receptor and undergo conformational change, which leads to a
Vikesh Chhabria' \& Steve Beeton*,1

'School of Pharmacy \& Biomedical Sciences, University of Central Lancashire, Preston, Lancashire, PR1 2HE, UK

*Author for correspondence: sbeeton@uclan.ac.uk 
pore spanning across the membrane [1]. There are two families of PFTs, classified according to their secondary structure. There are $\alpha$-PFTs and $\beta$-PFTs; majority of PFTs are of the $\beta$-family of toxins [5]. Toxins bind to various targets on the cellular membrane, some are specific for proteins such as $\alpha$-hemolysin and the other bind to lipids on the membrane (Figure 1). One such example is a group that falls under the $\beta$-PFTs, known as cholesterol-dependent cytolysins. An example of this type of PFTs is streptolysin-O. This toxin binds to cholesterol embedded in lipid bilayers present in some mammalian cells [6].

\section{Current treatment \& application of nanosponges}

Treatment options for sepsis include antibiotics, surgical drainage of infected fluids, blood transfusion, organ replacement and steroids. Most of these treatment strategies reduce the inflammatory response [7]. The firstline treatment would be intravenous administration of antibiotics. However, due to the high rates of antibiotic resistance, sepsis and other fatal conditions caused by these bacteria are becoming difficult to treat $[8]$. Therefore, this research aims to synthesize a biomimetic biosponge known as a 'nanosponge' that has the ability to absorb PFTs and therefore, in turn, could reduce the incidence and severity of exotoxin-related sepsis. Nanosponges are a nanoparticle constructed with a polymeric core (poly[D,L-lactic-co-glycolic acid] [PLGA]) enveloped by, in this case, RBC membranes. Each component of the nanosponge has a significant property. The RBC membrane contains surface properties that allow absorption of PFTs. Its highly flexible structure enables it to pass through narrow capillary networks [9]. By coating it with a mammalian erythrocyte membrane, the nanosponge is nonimmunogenic even if released back into the same mammalian species.

\section{Materials \& methods}

All chemicals were analytical grade and purchased from Fisher Scientific UK Ltd (Loughborough, UK), Sigma-Aldrich Ltd (Dorset, UK), Diagnostic Reagents Ltd (Oxfordshire, UK) and Electron Microscopy Sciences (PA, USA). Ovine and porcine blood was collected from an abattoir (William Taylor and Sons, Lancashire, UK). Murine blood was collected from the university of Central Lancashire Laboratory (Lancashire, UK). Leporine blood was purchased from TCS biosciences (Botolph claydon, Buckingham, UK).

\section{Development \& characterization of ovine erythrocyte ghosts}

Erythrocyte ghosts were produced using a ghosting protocol [10]. Erythrocytes were washed three-times with isotonic $(\mathrm{pH}$ 7.2) phosphate buffered saline (PBS) and centrifuged at $500 \times g$ to produce a concentrated erythrocyte suspension. The washing allowed removal of the buffy coat (platelets and white blood cells) and plasma. This erythrocyte suspension was subjected to hypotonic lysis, which allowed the release of free cellular hemoglobin $(\mathrm{Hb})$ from the erythrocyte. The lysed erythrocyte suspension was left on ice for $20 \mathrm{~min}$. The suspension was then centrifuged at $22,000 \times g$ for $10 \mathrm{~min}$ at $4^{\circ} \mathrm{C}$. The $\mathrm{Hb}$ present in the supernatant was washed with isotonic PBS and then recentrifuged at $22,000 \times g$ for $10 \mathrm{~min}$. A pink pellet was formed at the end of the tube, which was the erythrocyte ghosts. The erythrocyte ghosts have the property to reseal; however, the reason is unknown. The resealed erythrocyte ghosts were collected and examined using a previously published scanning electron microscopy protocol with slight modifications [11]. The modifications to the protocol were the concentration of the fixatives used and the duration of fixation. The erythrocyte ghosts were fixed in 3\% glutaraldehyde for $3 \mathrm{~h}$ and then in $2 \%$ osmium tetroxide for $2 \mathrm{~h}$. The dried pellet was fixed onto a carbon stub and coated with gold. The ovine erythrocyte ghosts were observed under a Quanta 200 scanning electron microscope (OR, USA).

\section{Synthesis of ovine erythrocyte vesicles}

The ovine erythrocyte ghosts were reconstituted in $3 \mathrm{ml}$ of isotonic PBS ( $\mathrm{pH}$ 7.2) and sonicated using a VWR (Leicestershire, England) sonicating water bath $(50-60 \mathrm{~Hz})$ for $20 \mathrm{~min}$ to produce erythrocyte vesicles of nonuniform size $(1.0-2.1 \mu \mathrm{m})$. The resulting vesicles were serially extruded through a $400-\mathrm{nm}$ and then a $100-\mathrm{nm}$ polycarbonate membrane 13 -times at $20^{\circ} \mathrm{C}$ (Figure 2), using a Avestin lipofast extruder (Weinheimer, Mannheim, Germany), which developed vesicles of uniform size (Figure 3B). The erythrocyte vesicles were characterized for size and polydispersity in isotonic $\mathrm{PBS}(\mathrm{pH} 7.2)$ at $37^{\circ} \mathrm{C}$ using a Malvern Nano-zs zetasizer (Malvern, Worcestershire, UK) [12].

\section{Production of PLGA polymeric core}

The PLGA polymeric cores were prepared using a solvent displacement process [13]. About $1 \mathrm{ml}$ of a $1 \mathrm{mg} /$ $\mathrm{ml}$ PLGA solution was prepared in acetone (organic solvent) and added dropwise to $3 \mathrm{ml}$ of distilled water. This solution was stirred for $4 \mathrm{~h}$ at $20^{\circ} \mathrm{C}$ to allow the organic phase to evaporate. The prepared PLGA cores were precipitated using a Merck Millipore filter with a $10-\mathrm{kDa}$ molecular weight cutoff. The PLGA cores were washed three-times with isotonic PBS to filter out the organic solvent. The precipitated PLGA nanoparticles were reconstituted in $1 \mathrm{ml}$ PBS. 


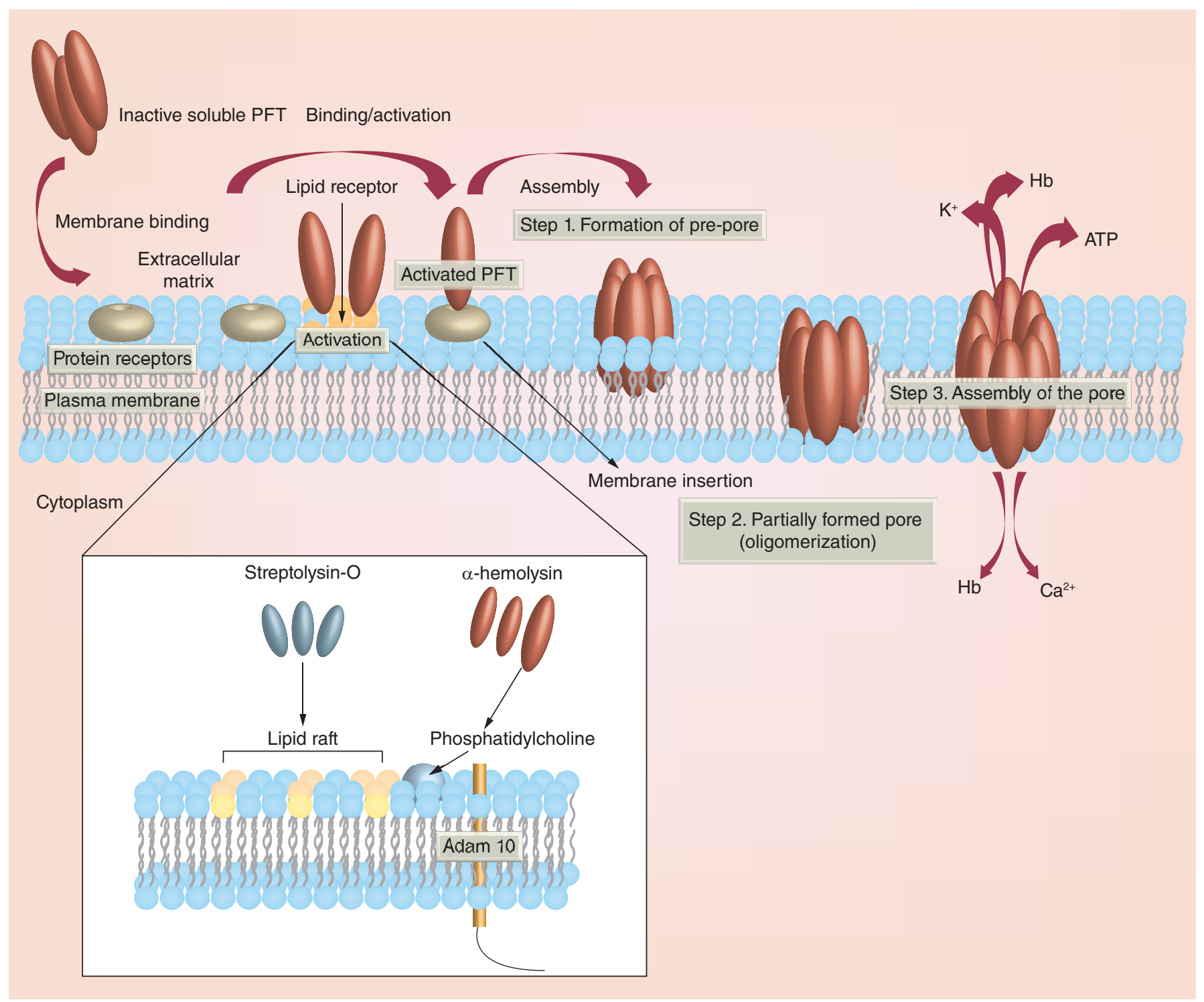

Figure 1. Mechanisms of pore formation by pore-forming exotoxins. Diagrammatic representation of pore formation. Poreforming exotoxins (PFTs), specific for certain receptors, recognize the target site on the membrane, which are either lipids (phosphatidylcholine, cholesterol and sphingomyelin) or proteins (ADAM 10). Upon binding the toxin starts oligomerizing. For $\beta$-PFTs, oligomerization occurs on the surface of the membrane producing a structure known as a prepore, which eventually produces a pore that spans across the membrane. $\beta$-PFTs like streptolysin-O (cholesterol-dependent cytolysin) have high affinity toward the lipid raft domain embedded in cholesterol. Another $\beta$-PFTs $\alpha$-hemolysin is specific for a protein known as ADAM 10 and also a phospholipid group called phosphatidylcholine.

\section{Synthesis of nanosponges}

The erythrocyte vesicles and the PLGA polymeric cores were added in equal volumes and extruded 13 -times through a 100 -nm polycarbonate membrane at $20^{\circ} \mathrm{C}$. The mechanical force of extrusion allowed fusion of the erythrocyte vesicles with the PLGA nanoparticle, synthesizing a nanoparticle with a lipid coating and a PLGA polymeric core [12]. Nanosponges were characterized using standard operating protocols [9]. Nanosponges $(1 \mathrm{mg} / \mathrm{ml})$ were characterized for size and polydispersity in isotonic $\mathrm{PBS}\left(\mathrm{pH} 7.2\right.$ ) at $37^{\circ} \mathrm{C}$ using a Malvern Nano-zs zetasizer.

\section{Toxin lysis assay}

Streptolysin-O was assayed for hemolysis against four different species of mammalian blood (porcine, leporine, ovine and murine). Parameters were selected on the basis of a recent publication, with modifications [3]. Increasing concentrations of streptolysin-O were added to a $500 \mu \mathrm{l} 2 \%(\mathrm{v} / \mathrm{v})$ erythrocyte suspension. 


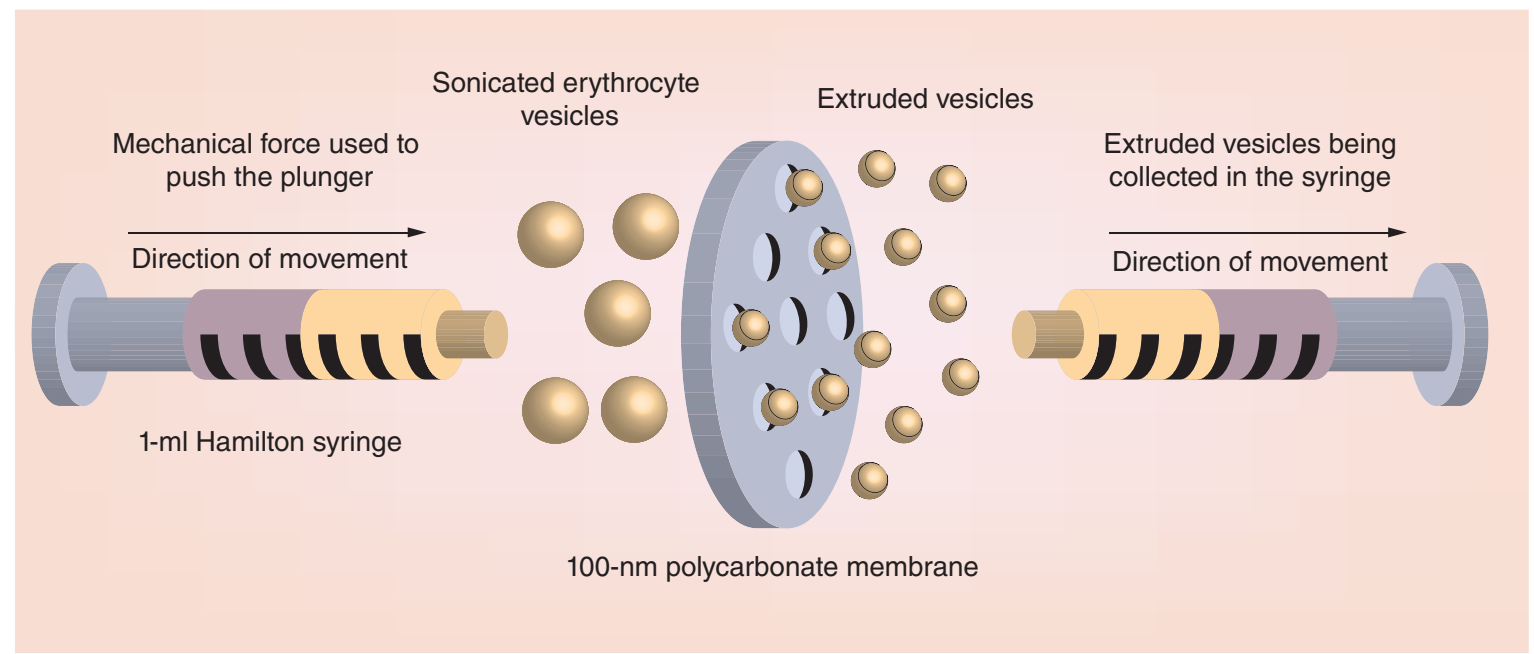

Figure 2. Mechanism of extrusion. A diagrammatic representation of sonicated vesicles being extruded through a $100-\mathrm{nm}$ polycarbonate membrane. The vesicles are extruded using the mechanical force generated by the push of the syringe. This mechanical energy forces the vesicles through the $100-\mathrm{nm}$ membrane synthesizing vesicles of uniform size.

The mixed suspension containing toxin and erythrocytes were incubated at $37^{\circ} \mathrm{C}$ for $30 \mathrm{~min}$. These were then centrifuged and $20 \mu \mathrm{l}$ of the supernatant was added to $4 \mathrm{ml}$ of Drabkins reagent (Diagnostic reagents Ltd, Oxfordshire, UK). The optical density was then recorded at $540 \mathrm{~nm}$ using a Jenway spectrophotometer (Bibby Scientific Ltd, Staffordshire, UK). The $\mathrm{Hb}$ was estimated using a bovine $\mathrm{Hb}$ standard curve (Supplementary Figure 1). The concentration of $\mathrm{Hb}$ released was represented as a percentage of the total $\mathrm{Hb}$ present in a $2 \%(\mathrm{v} / \mathrm{v})$ erythrocyte suspension, as the $\mathrm{Hb}$ concentration differs between animals. The assay has a detection range between 250 and $0.2 \mathrm{mg} / \mathrm{ml}$ streptolysin-O. The Drabkins reagent also had a detection range of $0.01-11.2 \mathrm{~g} / \mathrm{dl}$.

\section{Toxin absorption assays}

Testing the efficacy of erythrocyte ghosts as a toxin-absorbing system

Absorption of streptolysin-O by erythrocyte ghosts was tested using a novel assay. Washed ovine blood was diluted to make a $2 \%(\mathrm{v} / \mathrm{v})$ erythrocyte suspension, 2\% (v/v) erythrocyte ghost suspension and a 2\% $(\mathrm{v} / \mathrm{v})$ mixed (ghosts and erythrocytes) RBC suspension (Supplementary Figure 2). The 2\% erythrocyte ghost suspension was made by adding 2 parts of washed blood to 98 parts of hypotonic buffer $(9.64 \mathrm{mM} \mathrm{NaCl}$, $1.20 \mathrm{mM} \mathrm{KH} \mathrm{PO}_{4}, 1 \mathrm{mM}$ EDTA and $3.61 \mathrm{mM}$ $\mathrm{Na}_{2} \mathrm{HPO}_{4}$ ). This buffer was used to lyse erythrocytes to synthesize the erythrocyte ghosts. The erythrocyte ghost pellet was washed three-times with isotonic PBS and then reconstituted in it. The $2 \%$ mixed erythrocyte suspension was made by adding one part of the $2 \%$ erythrocyte suspension to one part of the $2 \%$ erythro- cyte ghost suspension. About $1230 \mathrm{ng} / \mathrm{ml}$ (estimated human physiological concentration) of streptolysin-O was added to the three suspensions and incubated in a water bath at $37^{\circ} \mathrm{C}$ for $30 \mathrm{~min}$. The suspensions were then centrifuged for 5 min at $900 \times g$ in an Eppendorf $540 \mathrm{R}$ microcentrifuge (Arlington business park, Stevenage, UK) at $4^{\circ} \mathrm{C}$. The concentration of $\mathrm{Hb}$ was measured by adding $20 \mu \mathrm{l}$ of the supernatant to $4 \mathrm{ml}$ Drabkins reagent and the solution was allowed to stand for $15 \mathrm{~min}$. The optical density was read at $540 \mathrm{~nm}(\mathrm{Hb} \mathrm{A})$. About $250 \mu \mathrm{l}$ of the supernatant was aspirated from each system and added to a separate $2 \% \mathrm{RBC}$ suspension, which was incubated for $30 \mathrm{~min}$ at $37^{\circ} \mathrm{C}$. The tubes were then centrifuged at $4^{\circ} \mathrm{C}$ for $5 \mathrm{~min}$ at $900 \times \mathrm{g}$. About $20 \mu \mathrm{l}$ of the supernatant is added to $4 \mathrm{ml}$ of Drabkins reagent and was allowed to stand for $15 \mathrm{~min}$. The optical density was then read at $540 \mathrm{~nm}(\mathrm{Hb} \mathrm{B})$. The concentration of $\mathrm{Hb}$ released by unbound toxin was measured by subtracting $\mathrm{Hb} \mathrm{A}$ from $\mathrm{Hb} \mathrm{B}$. This procedure was repeated with $0.1 \%$ Triton $^{\mathrm{TM}}$ X-100 (v/v) from Sigma-Aldrich (Dorset, UK) as a positive control.

Testing the efficacy of nanosponges as a toxinabsorbing system

Toxin absorption by nanosponges was tested using a published method [9]. This method was optimized to achieve desired experimental conditions. About $50 \mu \mathrm{l}$ of a $1230 \mathrm{ng} / \mathrm{ml}$ (sheep physiological concentration at which $50 \%$ of the erythrocytes were lysed) streptolysinO solution was added to four test systems: streptolysin$\mathrm{O}$ and 2\% erythrocyte suspension; streptolysin-O, erythrocyte vesicles and $2 \%$ erythrocyte suspension; streptolysin-O, PLGA polymer and $2 \%$ erythrocyte 
suspension; and streptolysin- $\mathrm{O}$, ovine nanosponges and $2 \%$ erythrocyte suspension. The total volume in these four different systems was $550 \mu$ l. These four systems were then incubated at 37 and $40^{\circ} \mathrm{C}$ which emulated the elevated body temperature during sepsis [14]. The suspensions were then centrifuged for $5 \mathrm{~min}$ at $900 \times g$. About $20 \mu \mathrm{l}$ of the supernatant was added to $4 \mathrm{ml}$ of Drabkins reagent and allowed to stand for $15 \mathrm{~min}$. The optical density was then read at $540 \mathrm{~nm}$.

\section{Statistical analysis}

Significance denoted, by an asterisk, was determined by a paired $t$-test using SPSS 22.0 (IBM, Portsmouth, $\mathrm{UK})$.

\section{Results}

Morphology of porcine erythrocytes \& ghosts

The erythrocyte ghosts were characterized using scanning electron microscopy. The erythrocyte ghost pellet was fixed in 3\% glutaraldehyde followed by fixation in 2\% osmium tetroxide (Supplementary Figure 3 ).

\section{Characterization of ovine erythrocyte vesicles}

The nanosponges were synthesized by fusing the PLGA core with the erythrocyte vesicles. Prior to fusion, the erythrocyte vesicles were measured for size and polydispersity. The vesicles were produced by sonication followed by extrusion through 400 - and $100-n m$ polycarbonate membranes. Sonication times did have a significant impact on polydispersity index (PDI) and size of the erythrocyte vesicles. Vesicles sonicated for $21 \mathrm{~min}$ had a PDI of $0.78( \pm 0.04)$ compared with vesicles sonicated for $14 \mathrm{~min}$, which had a PDI of $0.33( \pm$ 0.04 ) (Figure $3 \mathrm{~A}$ ). Vesicles sonicated for $21 \mathrm{~min}$ had a smaller average size of $1041( \pm 445) \mathrm{nm}$ compared with vesicles sonicated for $14 \mathrm{~min}$ (Figure 3B). Sonicated vesicles were serially extruded, which decreased the PDI to $0.13( \pm 0.01)$ (Figure $3 \mathrm{~A})$ and the size to $181( \pm 39)$ $\mathrm{nm}$ (Figure $3 \mathrm{~B})$. The nanosponges were also characterized using dynamic light scattering (DLS) for size and PDI (Supplementary Figure 4). The nanosponges had a size of $185 \mathrm{~nm}( \pm 5.0)$ with a PDI of 0.134 and a $\zeta$ potential of $-10.5 \mathrm{mV}( \pm 7.4)$.

\section{Streptolysin-O hemolytic assay}

Streptolysin-O was assayed against four different species of mammalian blood. A 2\% (v/v) erythrocyte suspension was prepared, to which increasing concentrations of streptolysin- $\mathrm{O}$ were added. The suspensions were incubated at $37^{\circ} \mathrm{C}$ for $30 \mathrm{~min}$. The suspensions were then centrifuged and the supernatant was read for $\mathrm{Hb}$ concentration at $540 \mathrm{~nm}$. The measured $\mathrm{Hb}$ was estimated using a bovine $\mathrm{Hb}$ standard curve (Supplementary Figure 1). Since each animal blood has a different amount of $\mathrm{Hb}$ present in their system, hemolysis was calculated as a percentage of the total amount of $\mathrm{Hb}$ present in a $2 \%$ erythrocyte suspension. According to Figure 4, at high concentrations of streptolysin$\mathrm{O}$, the $2 \%$ ovine erythrocyte suspension was most susceptible to lysis by streptolysin-O toxin as $2000 \mathrm{ng} / \mathrm{ml}$ streptolysin-O released $80.08 \%( \pm 1.40) \mathrm{Hb}$ compared with the other species of blood. At lower concentrations of streptolysin-O (250-1250 ng/ml), murine erythrocytes are more susceptible to streptolysin-O lysis, compared with ovine erythrocytes.

\section{Streptolysin-O absorption assays}

Streptolysin-O absorption was tested across lipid and polymeric systems which when fused via extrusion developed into nanosponges. Figure $5 \mathrm{~A}$ shows that ovine erythrocyte ghosts had the ability to absorb streptolysin-O. This test involved the addition of supernatant containing unbound toxin to a $2 \%$ erythrocyte suspension. This was indicated by the degree of hemolysis. The system that contained $2 \%$ ovine RBC had the highest concentration of $\mathrm{Hb}$ release at $0.45 \mathrm{~g} /$ $\mathrm{dl}( \pm 0.05)$ compared with the system that contained $2 \%$ ghosts, which had the least $\mathrm{Hb}$ release at $0.30 \mathrm{~g} /$ $\mathrm{dl}( \pm 0.03)$. The system that contained a mixture of the ovine RBC and the ghost had nearly half the $\mathrm{Hb}$ release of the $2 \%$ ovine $\mathrm{RBC}$ system $(0.36 \mathrm{~g} / \mathrm{dl}$, \pm 0.03 ). These results were compared with a positive control, which involved the addition of $0.1 \%$ Triton $\mathrm{X}-100$ to the three different suspensions. This suspension has the ability to solubilize lipids, thereby releasing maximum amount of $\mathrm{Hb}$ from the erythrocytes and ghosts.

The ovine erythrocyte ghosts were sonicated to produce vesicles, which were extruded with the PLGA polymer to produce nanosponges. Figure 5B shows the efficacy of the nanosponge and its components to absorb streptolysin-O. This was tested by adding the streptolysin-O and $2 \%$ RBC to systems containing the PLGA polymer, vesicles and nanosponges, which were incubated at $37^{\circ} \mathrm{C}$ for $30 \mathrm{~min}$. Absorption was measured by concentration of $\mathrm{Hb}$ release (Supplementary Figure 5). The system that contained the nanosponges had the least $\mathrm{Hb}$ release at $0.005 \mathrm{~g} /$ $\mathrm{dl}( \pm 0.005)$ compared with the system containing just the toxin and erythrocytes $(0.23 \mathrm{~g} / \mathrm{dl}, \pm 0.01)$. The system that contained the vesicles was able to absorb the toxins similar to the ovine erythrocyte ghosts in the previous test, as it had nearly half the $\mathrm{Hb}$ release $(0.08 \mathrm{~g} / \mathrm{dl}, \pm 0.01)$, as opposed to the system containing just toxin and erythrocytes. A similar result was achieved when the four different systems were incubated at $40^{\circ} \mathrm{C}$ (estimated human body temperature during sepsis) as shown in Figure $5 \mathrm{C}$. 

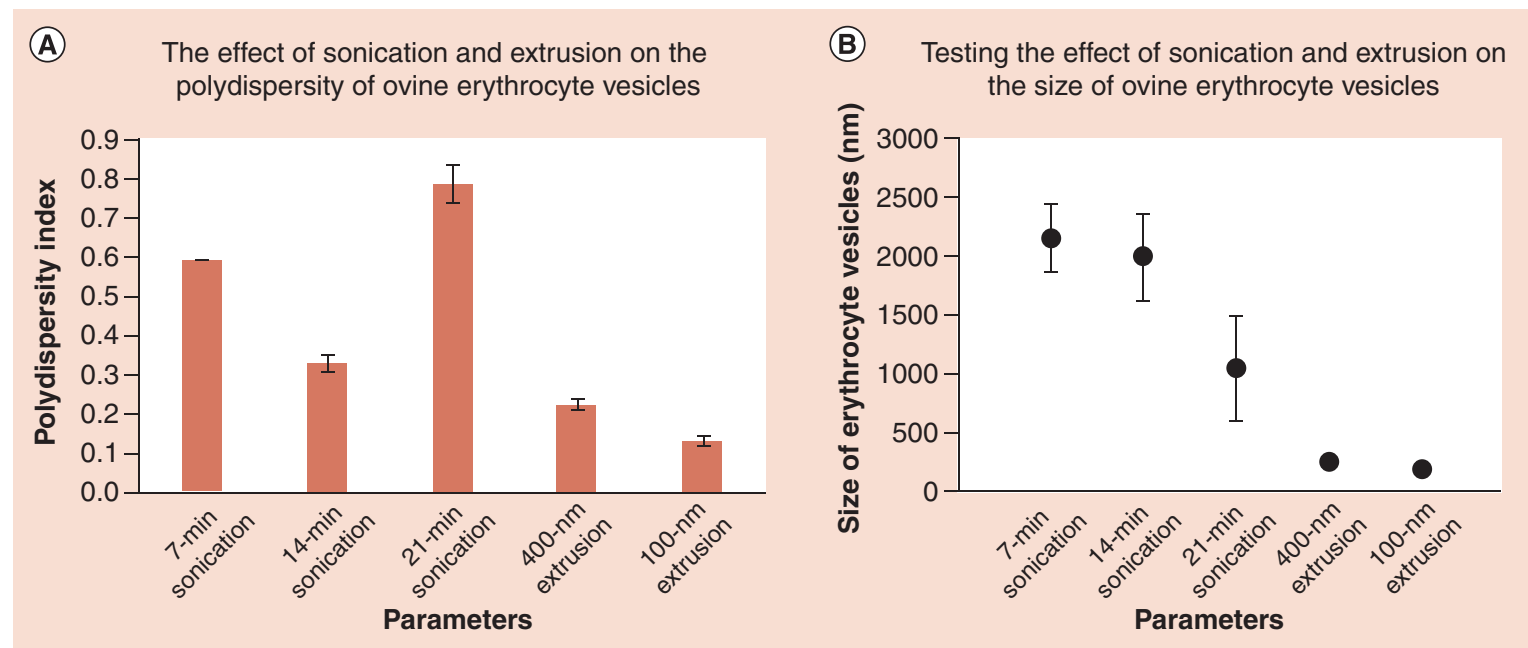

Figure 3. Characterization of ovine erythrocyte vesicles by measuring the size and polydispersity index using dynamic light scattering. (A) Measurement of polydispersity index of erythrocyte vesicles, which were sonicated for 7, 14 and 21 min followed by 400 and $100 \mathrm{~nm}$ extrusion. (B) Estimation of the size of erythrocyte vesicles that were sonicated for 7,14 and $21 \mathrm{~min}$ followed by 400 - and 100-nm extrusion. The ovine erythrocyte vesicles were produced by sonicating and extruding the erythrocyte ghosts at $25^{\circ} \mathrm{C}$. Error bars represent standard error of mean $(n=3)$.

The nanosponge and its components were lyophilized in $5 \%$ sucrose and stored at $2-4^{\circ} \mathrm{C}$ for 1 week. The nanosponge and its components were then reconstituted with PBS and tested for their efficacy to adsorb streptolysin-O (Figure 5D). Similar to the previous results the system that contained the nanosponge shows the least $\mathrm{Hb}$ release at $0.05 \mathrm{~g} / \mathrm{dl}( \pm 0.04)$.

\section{Nanosponge dose-dependent assay}

The ovine nanosponges $(1 \mathrm{mg} / \mathrm{ml})$ were added in increasing concentration to systems that contained $2 \%$ ovine erythrocytes and $1230 \mathrm{ng} / \mathrm{ml}$ streptolysin$\mathrm{O}$. The mixed suspensions were incubated at $40^{\circ} \mathrm{C}$ for $30 \mathrm{~min}$. The suspensions were then centrifuged and the supernatant was measured for concentration of $\mathrm{Hb}$ released. According to Figure 6, 10,000 ng/ml nanosponge absorbed $1230 \mathrm{ng} / \mathrm{ml}$ streptolysin-O as the concentration of $\mathrm{Hb}$ released is minimal $(0.009 \mathrm{~g} / \mathrm{dl}$, \pm 0.005 ). Comparatively, $2000 \mathrm{ng} / \mathrm{ml}$ nanosponge had the highest concentration of $\mathrm{Hb}$ released at $0.554 \mathrm{~g} / \mathrm{dl}$ $( \pm 0.03)$.

\section{Discussion}

The overall aim of the research was to study the adsorption of a bacterial PFT by previously developed biomimetic nanosponges [9], under temperature conditions that mimic sepsis in humans [14]. This study was conducted using ovine blood as an animal model. Previous studies have reported using ovine systems as a model for endotoxin-derived sepsis, as their blood components are similar to that of humans and they have similar physiological parameters [15]. All the experiments conducted in this study to prove toxin adsorption involve measurement of $\mathrm{Hb}$ released.

\section{Characterization of ovine erythrocyte vesicles}

The nanosponges were synthesized by fusing the PLGA core with erythrocyte vesicles. The ovine erythrocyte vesicles were characterized using two parameters (PDI and size) measured by the principles of dynamic light scattering. Sonication and extrusion were used to assess homogeneity among the vesicles. Figure 3A showed that sonication after $21 \mathrm{~min}$ developed polydispersed particles $(\mathrm{PDI}=0.78)$; however, vesicles sonicated for $21 \mathrm{~min}$ had a smaller average size of $1041 \mathrm{~nm}$ (Figure 3B). Extrusion of ovine erythrocyte vesicles produced homogeneous vesicles with a PDI of 0.13 and an average size of $181 \mathrm{~nm}$ (Figure 3B). Assessing homogeneity and size is significant as erythrocytes travel through capillaries and sinusoids. If the particle size is too large, this could lead to aggregation [16]. This will cause the erythrocyte vesicles to lose its function as a biomimetic nanoparticle. Reduction in size of ovine vesicles, due to extrusion and longer sonication times, was significant as it increases the surface area to volume ratio of the nanosponges (Supplementary Figure 6). Higher surface area to volume ratio leads to a higher rate of toxin adsorption [17]. Compared with ovine erythrocytes $(3.6 \mu \mathrm{m})$, the size of the nanosponge was recorded as $185 \mathrm{~nm}$ (Supplementary Figures $4 \& 7$ ). The surface area to volume ratio of ovine nanosponges is 19-times higher than ovine erythrocytes, which means that the ovine 




Figure 4. The effect of streptolysin-O on hemoglobin release from four different types of mammalian erythrocyte suspensions. Increasing concentrations of streptolysin-O were added to a $2 \%$ erythrocyte suspension made from leporine (rabbit $\Delta)$, murine (rat $\bullet$ ), porcine (pig $\bullet$ ) and ovine (sheep $\bullet$ ) blood. Total hemoglobin present in a $2 \%$ erythrocyte suspension: leporine $(2.67 \mathrm{~g} / \mathrm{dl}, \pm 0.07)$, murine $(1.87 \mathrm{~g} / \mathrm{dl}, \pm 0.07)$, ovine $(1.43 \mathrm{~g} / \mathrm{dl}, \pm 0.09)$ and porcine $(2.47 \mathrm{~g} / \mathrm{dl}, \pm 0.32)$. Error bars represent standard error of mean $(n=3)$. The assays were incubated at $37^{\circ} \mathrm{C}$ for $30 \mathrm{~min}$.

nanosponges will have more surface area than volume to adsorb streptolysin-O.

\section{Streptolysin-O hemolytic assay}

This study investigated the degree of streptolysin-O hemolysis against ovine, porcine, leporine and murine erythrocytes. Streptolysin-O is a pore-forming toxin secreted by group A streptococcus and is shown to bind specifically to cholesterol on the erythrocyte membrane [18]. Figure 4 showed that streptolysin-O was highly sensitive toward ovine erythrocytes, as $2000 \mathrm{ng} /$ $\mathrm{ml}$ of streptolysin-O released $80.08 \%( \pm 1.40)$ ovine $\mathrm{Hb}$ compared with other species of blood. According to a previously published study, ovine erythrocytes had a higher concentration of cholesterol present in the membrane [19]. At lower concentrations of streptolysin-O $(250-1250 \mathrm{ng} / \mathrm{ml})$, murine erythrocytes are more susceptible to streptolysin-O lysis, compared with ovine erythrocytes. The reason for this is unknown. Further tests may need to be employed in order to verify this result. This study suggested that ovine erythrocytes can be used as an animal model to develop biomimetic nanosponges as they showed the highest affin- ity toward streptolysin-O attack. An animal model has many advantages as it could aid in the development of new therapeutic platform; animal systems could be easily manipulated to achieve physiological conditions similar to that of human; and several clinical features in animals are similar to humans [20].

\section{Absorption of streptolysin-O by lipid \& polymeric systems}

This assay tested ovine nanosponge and its components for toxin adsorption. The $2 \%(\mathrm{v} / \mathrm{v})$ ghost suspension was examined by scanning electron microscopy and it showed the presence of ghosts (Supplementary Figure 3). The 2\% (v/v) ghost suspension absorbed most streptolysin-O, as the concentration of $\mathrm{Hb}$ released was the lowest compared with the other systems (Figure 5A). Recently published studies showed that production of erythrocyte ghosts does not lead to the loss of lipids and cholesterol from the membrane [11]. The scanning electron micrograph (Supplementary Figure 3) shows morphologically intact erythrocyte ghosts, which could signify that cholesterol was present in the membrane. This result may be further understood as 


\section{(A)}

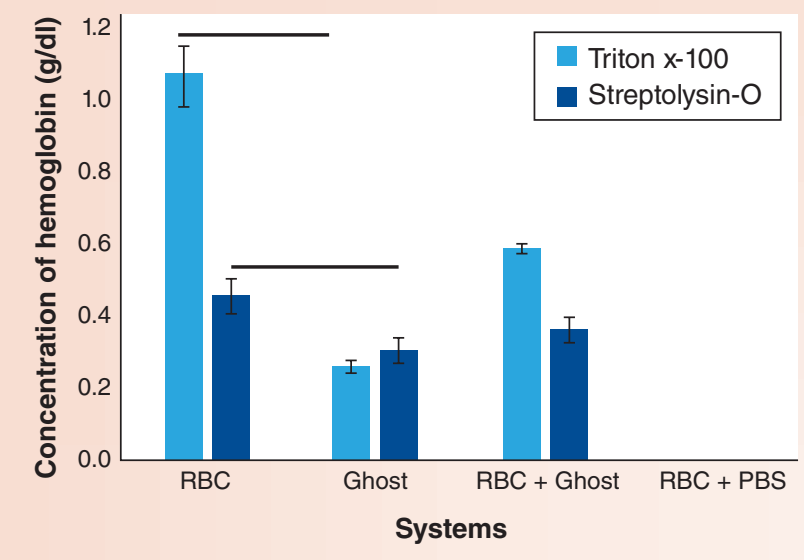

(C)

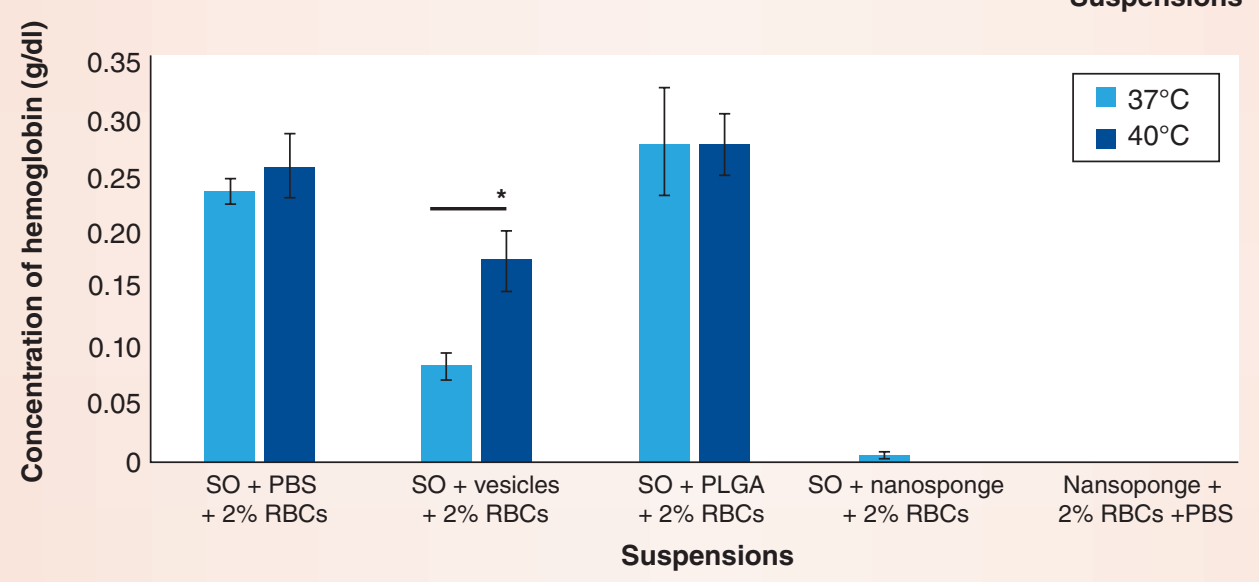

(D)

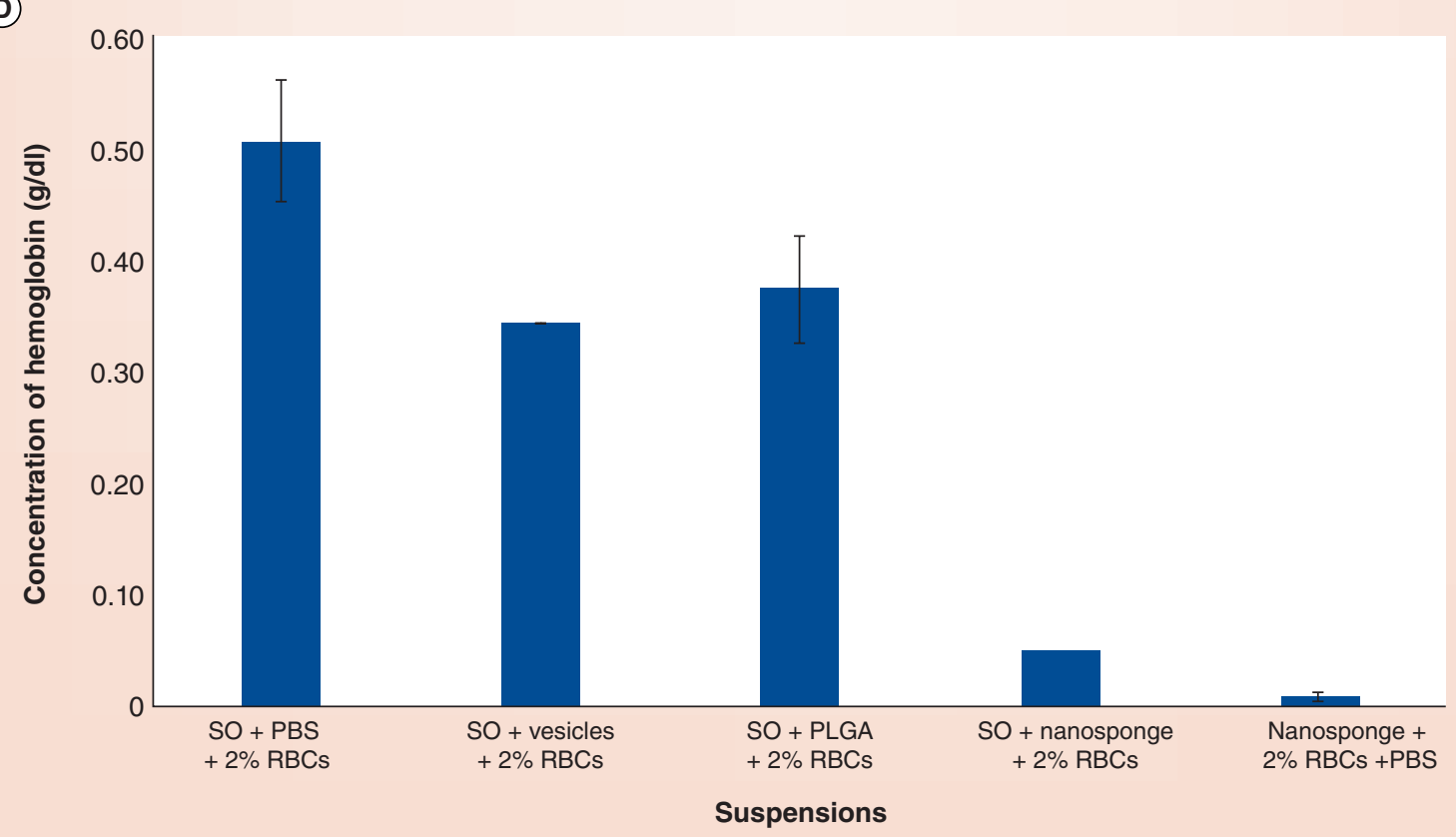

(B)



the erythrocyte ghosts, according to Figure 5A, have lesterol may be present in the membrane. However, adsorbed streptolysin-O. Thus, it indicates that cho- this could be tested by measuring the concentration of 
Figure 5. Testing absorption of $1230 \mathrm{ng} / \mathrm{ml}$ streptolysin-O by different lipid and polymeric systems, which make up the nanosponge (see facing page). (A) Testing the efficacy of ovine erythrocyte ghosts as a toxin-absorbing system, incubated at $37^{\circ} \mathrm{C}$. The erythrocyte ghosts were made from $1 \mathrm{ml}$ of whole blood. (B) Testing the nanosponge (1 mg/ml) and its components for absorption of streptolysin-O, incubated at $37^{\circ} \mathrm{C}$ for $30 \mathrm{~min}$. (C) Testing the nanosponge (1 $\mathrm{mg} / \mathrm{ml}$ ) and its components for absorption of streptolysin- $\mathrm{O}$, incubated at 37 and $40^{\circ} \mathrm{C}$ (human body temperature during sepsis) for $30 \mathrm{~min}$. (D) Testing reconstituted nanosponge $(1 \mathrm{mg} / \mathrm{ml})$ and its components for absorption of streptolysin-O, incubated at $40^{\circ} \mathrm{C}$. Error bars represent standard error of mean $(n=3)$.

$* \mathrm{p} \leq 0.05 ; * * \mathrm{p} \leq 0.01 ; * * * \mathrm{p} \leq 0.001$.

cholesterol present in the membrane of ovine erythrocytes and ghosts, by using a cholesterol esterase colorimetric assay [21]. This statement is further enhanced as nanosponges in Figure $5 \mathrm{~B}$ have managed to adsorb streptolysin-O. This could mean that during synthesis of the nanosponge, cholesterol was not lost.

Fusion of ovine erythrocyte vesicles and the PLGA polymer developed ovine nanosponges. Figure $5 B$ showed the efficacy of the nanosponge and its components to adsorb streptolysin-O. The system containing the nanosponges had the least $\mathrm{Hb}$ release compared with the system containing just the toxin and erythrocytes. The ovine erythrocyte vesicles were also able to absorb streptolysin-O as they had nearly half the $\mathrm{Hb}$ release, as opposed to the system containing just toxin and erythrocytes. This could be due to the surface area to volume ratio, as the nanosponges have a greater surface area to volume ratio (Supplementary Figure 6) [17]. Moreover, a published study suggests that due to the presence of PLGA in the core, it has the ability to lock the toxin compared with erythrocyte vesicles [9].

The same study was conducted at $40^{\circ} \mathrm{C}$ which is an estimation of the elevated body temperature during sepsis [14]. Figure $5 \mathrm{C}$ showed that there was no difference in the concentration of $\mathrm{Hb}$ released by three of the systems, incubated at 37 and $40^{\circ} \mathrm{C}$. Incubated

at $40^{\circ} \mathrm{C}$, the system that contained the nanosponges had adsorbed the streptolysin-O as there was no $\mathrm{Hb}$ released compared with the system at $37^{\circ} \mathrm{C}$. This may indicate that the nanosponges have a better absorption efficiency at $40^{\circ} \mathrm{C}$. This could be justified as the physiological body temperature of sheep is $39^{\circ} \mathrm{C}$ [22] This may also highlight the reason for statistical significance between the systems that contained vesicles (Figure 3C).

A similar study was conducted with reconstituted nanosponges and similar results were obtained. Figure 5D showed that ovine nanosponges had adsorbed all the streptolysin- $\mathrm{O}$ as there was no $\mathrm{Hb}$ released. This also showed that nanosponges have the ability to adsorb toxins after a week of being in a freeze-dried state. This may suggest that the nanosponges are fairly stable in a lyophilized state. A dose-dependent study was conducted to identify the concentration required to adsorb streptolysin-O at $40^{\circ} \mathrm{C}$. Figure 6 indicated that $10,000 \mathrm{ng} / \mathrm{ml}$ ovine nanosponges completely adsorb streptolysin-O, as there was no Hb released.

\section{Conclusion}

Ovine erythrocytes and erythrocyte ghosts are susceptible to streptolysin-O hemolysis, thereby making sheep an appropriate model to develop nanosponges.

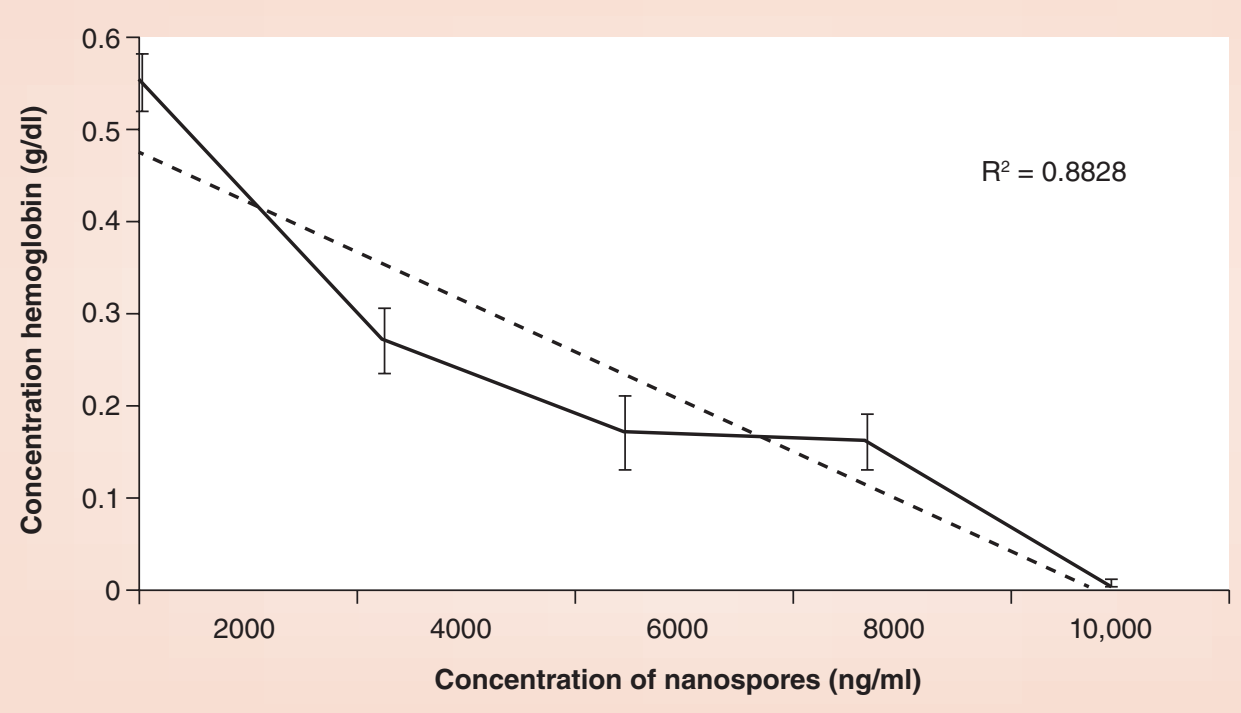

Figure 6. Concentrations at which, nanosponges can adsorb $1230 \mathrm{ng} / \mathrm{ml}$ streptolysin-O. Increasing concentrations of ovine nanosponges were added to $1230 \mathrm{ng} / \mathrm{ml}$ streptolysin- $\mathrm{O}$ and incubated at $40^{\circ} \mathrm{C}$ for $30 \mathrm{~min}$. Error bars represent standard error of mean $(n=3) . R^{2}=0.882$. 
Fusion of the erythrocyte vesicles with PLGA polymer forms the basis for a biomimetic nanosponge. This process is achieved by extrusion, which develops monodispersed nanosponges. Ovine nanosponges have the ability to absorb streptolysin-O at human body temperature, especially under conditions that mimic sepsis in humans. Reconstituted nanosponges from a lyophilized state retain their ability to adsorb streptolysin-O. The results obtained from this study identify ovine nanosponges as a potential novel therapeutic model to test the absorption of cholesterol-binding toxins. Further work is required to understand the role that PLGA plays in adsorption; moreover, specificity for toxin absorption could be increased by addition of external cholesterol into the membrane.

\section{Future perspective}

The use of cellular membranes as biomimetic therapeutic agent is a novel field of nanomedicine. Current research has shown that the field is limited to erythrocyte membranes. Over 5-10 years, the field may evolve to stable biomimetic formulations, such as platelets or white blood cell membranes for drug delivery and toxin adsorption. This biomimetic platform may also evolve into a diagnostic tool to test the presence of exotoxins in bodily fluids.

\section{Acknowledgements}

The authors would sincerely like to thank the University of
Central Lancashire for theory technical support toward this research. The authors would also like to acknowledge T Sen for his guidance and support in writing this paper.

\section{Supplementary data}

To view the supplementary data that accompany this paper please visit the journal website at: www.futuremedicine.com/ doi/full/10.2217/nnm-2016-0180

Financial \& competing interests disclosure

The authors have no relevant affiliations or financial involvement with any organization or entity with a financial interest in or financial conflict with the subject matter or materials discussed in the manuscript. This includes employment, consultancies, honoraria, stock ownership or options, expert testimony, grants or patents received or pending, or royalties.

No writing assistance was utilized in the production of this manuscript.

Ethical conduct of research

The ethical board at the University of Central Lancashire reviewed ethical approval for this research. The authors state that they have obtained appropriate institutional review board approval or have followed the principles outlined in the Declaration of Helsinki for all human or animal experimental investigations. In addition, for investigations involving human subjects, informed consent has been obtained from the participants involved.

\section{Executive summary}

\section{Sonication followed by extrusion develops monodispersed nanoparticles}

- Ovine erythrocyte vesicles were formed by sonication followed by extrusion, which produced monodispersed suspensions of ovine erythrocyte vesicles.

Hemolysis of different species of animal blood

- Ovine, porcine, murine and leporine blood showed that they were susceptible to streptolysin-O attack, measured by the concentration of hemoglobin released.

- Ovine erythrocytes were shown to be more specific for streptolysin-O binding, as the toxin was able to lyse ovine erythrocytes, which lead to the highest concentration of hemoglobin being released.

Ovine nanosponge form a good therapeutic model to test absorption of streptolysin-O

- Hemolysis assays identified ovine erythrocyte vesicles as an excellent lipid system to test toxin adsorption.

- Fusion of ovine erythrocyte vesicles with poly(D,L-lactic-co-glycolic acid) developed ovine nanosponges, which had the ability to adsorb streptolysin-O at human body temperature and in temperature conditions that mimic sepsis in humans.

- Reconstituted nanosponges from a lyophilized state retain their ability to adsorb streptolysin-O.

Dose-dependent assay

- Ovine nanosponges at a concentration of $10,000 \mathrm{ng} / \mathrm{ml}$ were able to adsorb $1230 \mathrm{ng} / \mathrm{ml}$ streptolysin-O.

\section{References}

1 Los FCO, Randis TM, Aroian RV, Ratner AJ. Role of poreforming toxins in bacterial infectious diseases. Microbiol. Mol. Biol. Rev. 77(2), 173-207 (2013).

2 Mcpherson D, Griffiths C, Williams M et al. Sepsisassociated mortality in England: an analysis of multiple cause of death data from 2001 to 2010 . BMJ 3(8), e002586 (2013).

3 Shewell LK, Harvey RM, Higgins MA et al. The cholesteroldependent cytolysins pneumolysin and streptolysin $\mathrm{O}$ require binding to red blood cell glycans for hemolytic activity. Proc. Natl Acad. Sci. USA 111(49), E5312-E5320 (2014). 
$4 \quad$ Fang RH, Luk BT, Hu CMJ, Zhang L. Engineered nanoparticles mimicking cell membranes for toxin neutralization. Adv. Drug Deliv. Rev. 90, 69-80 (2015).

5 Bonardi F, Nouwen N, Feringa BL, Driessen AJ. Protein conducting channels-mechanisms, structures and applications. Mol. Biosyst. 8(3), 709-719 (2012).

6 Keyel PA, Loultcheva L, Roth R et al. Streptolysin O clearance through sequestration into blebs that bud passively from the plasma membrane. J. Cell Sci. 124(14), 2414-2423 (2011).

7 Peters J, Cohen J. Sepsis. Medicine 41(11), 667-669 (2013).

8 Finch R, Hunter PA. Antibiotic resistance - action to promote new technologies: report of an $\mathrm{EU}$ Intergovernmental Conference held in Birmingham, UK, 12-13 December 2005. J. Antimicrob. Chemother. 58(Suppl. 1), i3-i22 (2006).

$9 \mathrm{Hu}$ CMJ, Fang RH, Copp J, Luk BT, Zhang L. A biomimetic nanosponge that absorbs pore-forming toxins. Nat. Nanotechnol. 8(5), 336-340 (2013).

10 Weed RI, Reed CF, Berg G. Is hemoglobin an essential structural component of human erythrocyte membranes?. J. Clin. Invest. 42(4), 581-588 (1963).

11 Kostić IT, Ilić VL, Đorđević VB et al.

Erythrocyte membranes from slaughterhouse blood as potential drug vehicles: isolation by gradual hypotonic hemolysis and biochemical and morphological characterization. Colloids Surf. B Biointerfaces 122, 250-259 (2014).

12 Hu CMJ, Zhang L, Aryal S, Cheung C, Fang RH, Zhang L. Erythrocyte membrane-camouflaged polymeric nanoparticles as a biomimetic delivery platform. Proc. Natl Acad. Sci. USA 108(27), 10980-10985 (2011).
13 Hu CMJ, Fang RH, Luk BT et al. 'Marker-of-self' functionalization of nanoscale particles through a topdown cellular membrane coating approach. Nanoscale 5(7), 2664-2668 (2013).

14 Lee BH, Inui D, Suh GY et al. Association of body temperature and antipyretic treatments with mortality of critically ill patients with and without sepsis: multi-centered prospective observational study. Crit. Care 16(1), 1-13 (2012).

15 Zarjou A, Agarwal A. Sepsis and acute kidney injury. J. Am. Soc. Nephrol. 22(6), 999-1006 (2011).

16 Kuo YC, Wu HC, Hoang D, Bentley WE, D’Souza WD, Raghavan SR. Colloidal properties of nanoerythrosomes derived from bovine red blood cells. Langmuir. 32(1), 171-179 (2016).

17 Jones DA. The important of surface area/volume ratio to the rate of oxygen uptake by red cells. J. Gen. Physiol. 74(5), 643-646 (1979).

18 Keyel PA, Roth R, Yokoyama WM, Heuser JE, Salter RD. Reduction of streptolysin O (SLO) pore-forming activity enhances inflammasome activation. Toxins (Basel) 5(6), 1105-1118 (2013).

19 Nelson GJ. Composition of neutral lipids from erythrocytes of common mammals. J. Lipid Res. 8(4), 374-379 (1967).

20 Fink MP. Animal models of sepsis. Virulence 5(1), 143-153 (2014).

21 Salè FO, Marchesini S, Fishman PH, Berra B. A sensitive enzymatic assay for determination of cholesterol in lipid extracts. Anal. Biochem. 142(2), 347-350 (1984).

22 Piccione G, Caola G, Refinetti R. Maturation of the daily body temperature rhythm in sheep and horse. J. Thermal Biol. 27(5), 333-336 (2002). 